\title{
Lessons learned from predictions of Solar Cycle 24
}

\author{
W. Dean Pesnell* \\ NASA Goddard Space Flight Center, Greenbelt, 20771 MD, USA
}

Received 1 June 2020 / Accepted 9 October 2020

\begin{abstract}
Solar Cycle 24 has almost faded and the activity of Solar Cycle 25 is appearing. We have learned much about predicting solar activity in Solar Cycle 24, especially with the data provided by SDO and STEREO. Many advances have come in the short-term predictions of solar flares and coronal mass ejections, which have benefited from applying machine learning techniques to the new data. The arrival times of coronal mass ejections is a mid-range prediction whose accuracy has been improving, mostly due to a steady flow of data from SoHO, STEREO, and SDO. Longer term (greater than a year) predictions of solar activity have benefited from helioseismic studies of the plasma flows in the Sun. While these studies have complicated the dynamo models by introducing more complex internal flow patterns, the models should become more robust with the added information. But predictions made long before a sunspot cycle begins still rely on precursors. The success of some categories of the predictions of Solar Cycle 24 will be examined. The predictions in successful categories should be emphasized in future work. The SODA polar field precursor method, which has accurately predicted the last three cycles, is shown for Solar Cycle 25. Shape functions for the sunspot number and F10.7 are presented. What type of data is needed to better understand the polar regions of the Sun, the source of the most accurate precursor of long-term solar activity, will be discussed.
\end{abstract}

Keywords: solar activity / forecasting methods

\section{Introduction}

Space Weather is driven by the periodic variation of the magnetic field at and above the solar surface. Dynamo motions of plasma within the convection zone generate that magnetic field. Once the magnetic field has erupted through the surface it can become organized into active regions. The magnetic field in and above these active regions will eventually be destroyed by either being converted into radiation and energetic particles by solar flares or by being ejected from the Sun in coronal mass ejections. The short-wavelength photons, energetic particles, and magnetic field emitted from the Sun by solar activity can affect satellites in orbit, radio-based communications, and terrestrial power grids.

Long-term predictions of the level of solar activity are needed by NASA to build spacecraft that will operate in the hostile environment of space. The predictions are needed to anticipate the space weather effects on space missions that can last two 11-year sunspot cycles. These predictions also test our understanding of the solar dynamo and the models used to understand the cause of flares and coronal mass ejections.

Ham radio operators are another consumer of solar cycle predictions. One ham radio operator lamented the inaccuracy

\footnotetext{
*Corresponding author: william.d.pesnell@nasa.gov
}

of the predictions of Solar Cycle 22, noting that the large range of available predictions meant no accurate prediction was possible (Weatherley, 1980). Solar Cycle 24 was somewhat of a disappointment to hams. It produced the worst propagation since the invention of $\mathrm{HF}$ radio! ${ }^{1} \mathrm{HF}$ radio uses frequencies between 3 and $30 \mathrm{MHz}$, which are strongly affected by ionospheric conditions.

Solar Cycle 24 started in December 2008, following a solar minimum that had lasted longer than average and reached record low levels of solar and geomagnetic activity (Solomon et al., 2010). This cycle has had a low level of activity, unlike the three similar Solar Cycles that preceded it but much like Solar Cycle 16 at the beginning of the 20th century.

Solar Cycle 24 may have had fewer sunspots than average but large events, such as the fast coronal mass ejection (CME) of 23 Jul 2012 (Russell et al., 2013) have still happened. Another unusual occurrence was active region 12,192, which formed in October 2014 as the largest active region since November 1990, reaching a peak area of $3300 \mu$-hems during its disk passage between 17 and 30 Oct 2014. It also produced $6 \mathrm{X}$-class and numerous M-class flares during its initial disk passage (Sun et al., 2015).

\footnotetext{
${ }^{1}$ https://forums.qrz.com/index.php?threads/the-hf-oracle-prognostication-for-solar-cycle-25.626433/
} 
Many predictions of the amplitude of Solar Cycle 24 appeared, even before the actual time of minimum (Pesnell, 2008, 2012, 2016). Based on the analysis below, this sunspot cycle generated more predictions than any of the previous three. Most of the predictions predict only the amplitude and time of the next solar maximum. Missing is the finer-grained time behavior, such as the timing of cycle extrema, which are usually found by examining not only the sunspot number record but also other measures of solar activity, such as the flare rate, emergence rate of magnetic flux, or areas of coronal holes, that may peak at different times of the sunspot cycle.

Solar Cycle 24 has now faded and Solar Cycle 25 is appearing. What did we learn from Solar Cycle 24 and how will that guide us into Solar Cycle 25.

\section{Data used}

We will use three data sets to explore predictions of solar cycle amplitude, two versions of the International Sunspot Number and the solar spectral irradiance at a wavelength of $10.7 \mathrm{~cm}$ (F10.7). The sunspot numbers are described in the next section.

F10.7 is a well-known index of solar activity that has been measured on a daily basis since 1947 Covington (1969) and Tapping \& Charrois (1994). F10.7 peaks at solar maximum and fades to around $60 \times 10^{-22} \mathrm{~W} \mathrm{~m}^{-2} \mathrm{~Hz}^{-1} \equiv 60 \mathrm{SFU}$ at solar minimum. The average of the annually-averaged peak value in the sunspot cycles since 1950 is $190 \pm 35$ SFU and the average of the top 15 daily values is $350 \pm 12$ SFU. It should be noted that the 15 highest daily values in Solar Cycle 23 averaged $275 \pm 15$ SFU. And 15 highest daily values in Solar Cycle 24 averaged $125 \pm 20$ SFU. So recent cycles are not well-represented by the earlier data.

Because it has a short latency of $24 \mathrm{~h}, \mathrm{~F} 10.7$ is often used as a proxy for solar activity in models of the thermosphere (to understand satellite drag) and ionosphere (for radio wave interference). Therefore, predictions of F10.7 are very useful to operators of satellites and the Space Weather community.

\section{Sunspot number and solar cycle shapes}

Mission designers and operators want solar cycle predictions today. The predictions don't have to be physically correct but they do have to be usable. They like F10.7 but want a standard variable. That standard is now $S$, which replaced $R_{Z}$ in July 2015. Both versions of the International Sunspot Number (Version 2, $S$, and Version $1, R_{Z}$ ) were downloaded from the SIDC website ${ }^{2}$ and are shown in Figure 1. Annually-averaged values of $S$ and $R_{Z}$ are shown since 1818, along with the ratio of the two versions. Although the current cycle has had a lower level of activity than any sunspot cycle since Solar Cycle 16 it is still an interesting cycle.

Predictions of Solar Cycle 24 used either $R_{Z}$ or F10.7 (converted to $R_{Z}$ with a linear correlation fit.) Predictions of Solar Cycle 25 have been reported in $R_{Z}, S$, and F10.7 (converted to $R_{Z}$ with a linear correlation fit.) The presence of two versions of the sunspot number adds to the uncertainty in

\footnotetext{
2 http://www.sidc.be/silso/
}

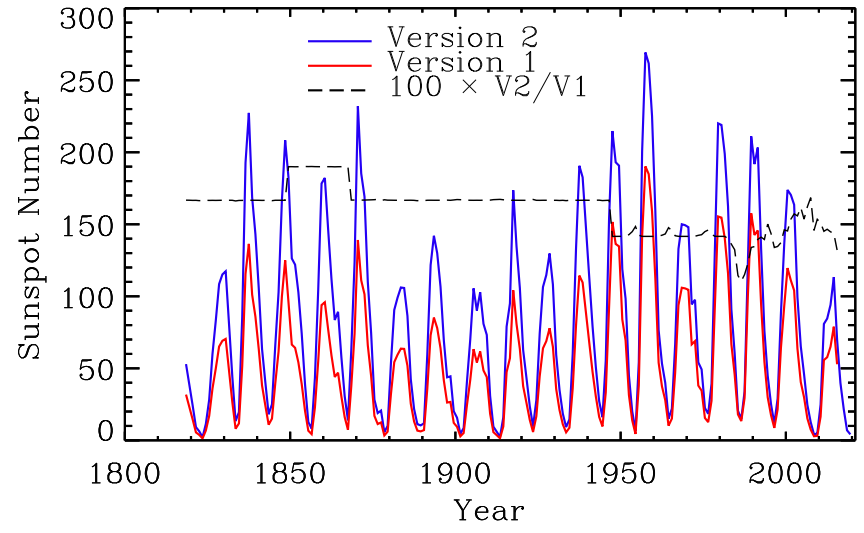

Fig. 1. The time dependence of annually-averaged $S$ (in blue) and $R_{Z}$ (in red) plotted as a function of time. Plotted in a dashed line to $100 \times S / R_{Z}$, showing how the major difference between the versions is a normalization, but recent values of the ratio have had a solar cycle dependence.

comparisons of the predictions within SC 25 and between SC 24 and SC 25.

\subsection{What is the sunspot number $(S)$ ?}

Sunspots are the longest existing quantitative record of solar activity. They have been recorded for some 400 years (about 35 cycles) including times of low to absent activity (such as the Maunder Minimum from 1645-1715) and the largest cycle on record (Solar Cycle 19, 1954.3-1964.8).

The sunspot number is the most common long-term index of solar activity created from telescope-aided visual counts of number of sunspots $\left(N_{s}\right)$ and the number of groups they form $\left(N_{g}\right)$. These counts are then combined to form the sunspot number. The form of the Version 1 sunspot number (also called the Wolf or Zurich sunspot number), $R_{Z}=k\left(10 N_{g}+N_{s}\right)$, was finalized by Wolf (1861) of Zurich Observatory. The $k$ is an observer-dependent quality factor that was set to 0.6 after Wolf died in 1893. Since 1981 the sunspot number has been produced at the World Data Center SILSO in Brussels from a worldwide network of over 85 stations. Daily values since 1818 are available from their archive, along with monthly means since 1749, and annual means since 1700. Data from before 1700 is more sporadic.

Between 2011 and 2015, a series of community-wide workshops resulted in the first re-calibration of the sunspot number (Clette et al., 2014; Clette \& Lefèvre, 2016). This new version of the sunspot number, $S=10 N_{g}+N_{s}$, has been served by SILSO since July 2015 and the original sunspot number was deprecated. The most important change was to remove the $k$, which scales the whole series upwards by about 1.6, with some time spans showing more complicated corrections. This is shown by the dashed line in Figure 1.

\subsection{Theoretical shape of sunspot number and F10.7}

A prediction of $S$ or F10.7 requires a shape function. Several authors have used the functional form

$$
S(t)=A \frac{t^{3}}{e^{(t / b)^{2}}-c}
$$



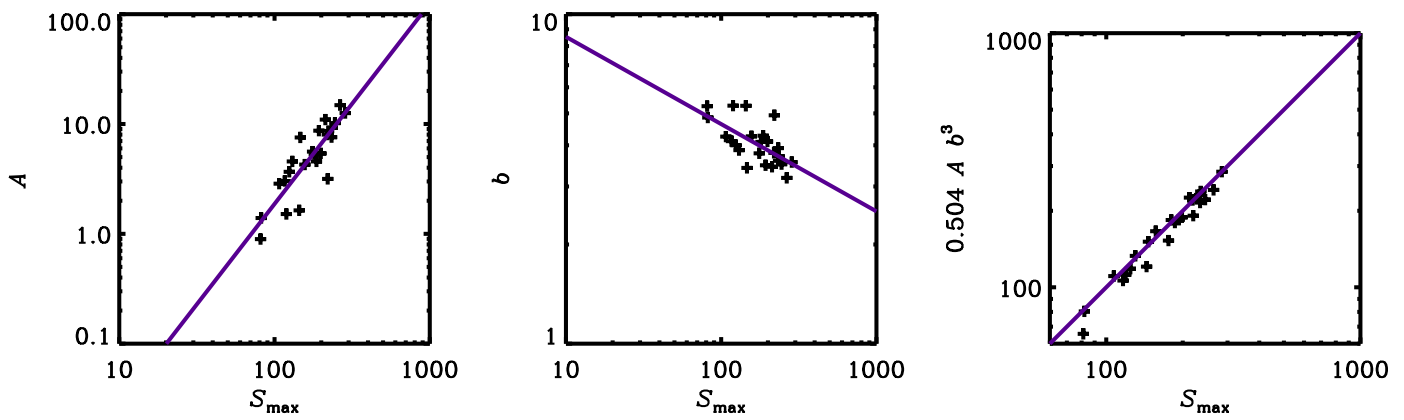

Fig. 2. The correlation fits used to derive the dependence of $A$ (left plot) and $b$ (middle panel) along with a demonstration that $S_{\max }=0.504 A b^{3}$ (drawn with a $45^{\circ}$ line.)

to represent the original sunspot number (Hathaway et al., 1994). Time is referenced to the time of solar minimum $\left(t_{\min }\right)$, which is not a free parameter in the fit. The use of a function that resembles a Debye function to represent $S$ can be traced to a Poisson distribution that omitted the $c$ from the denominator (Stewart \& Panofsky, 1938; Cook, 1949). It is timely to do this for $S$ as the recalibration changed the shape of several of the double-peaked cycles (Pesnell, 2018). The discrepancy in shape also reduced the agreement between the shape of $R_{Z}$ and F10.7. Shape functions for both will now be derived.

Assuming the shape function of equation (1) automatically provides several relationships. The shape parameter $c$ was included in a preliminary set of fits. That initial fitting was insensitive to the value of $c$, except for producing a few outliers that had $c>1$, which cannot be permitted. As a result, the value of $c$ was set to the average of those converged values $(c=0.74)$ and held constant for the final set of fits. Assuming $c$ is constant means that the time from minimum to maximum is linearly related to $b$ :

$$
t_{\max }-t_{\min }=1.081 b
$$

while the maximum of $S$ is

$$
S_{\max }=0.504 A b^{3} \text {. }
$$

The integral of the model sunspot number over a cycle is approximately

$$
\int_{0}^{\infty} S(t) \mathrm{d} t \approx 1.267 S_{\max } b,
$$

where the constant is the sum

$$
1.287 \approx \frac{0.992}{c} \sum_{k=1}^{\infty} \frac{c^{k}}{k^{2}} .
$$

We used the curvefit routine in IDL to fit annually-averaged values of $S$ in the available solar cycles with the function in equation (1). The times of solar minimum listed in Pesnell (2018) were used as the starting time of each cycle. An error of $2.6 \sqrt{S}$ was used in the fits. The fitted curves are shown in Figure 2, where $S$ is shown in black and the fits to each cycle are shown in colors. The fits capture most of the variation, making the theoretical fit useful for plotting predictions.

The values of $A$ and $b$ from each cycle where then fitted with power-laws of $S_{\max }$ to give smooth functions for use in

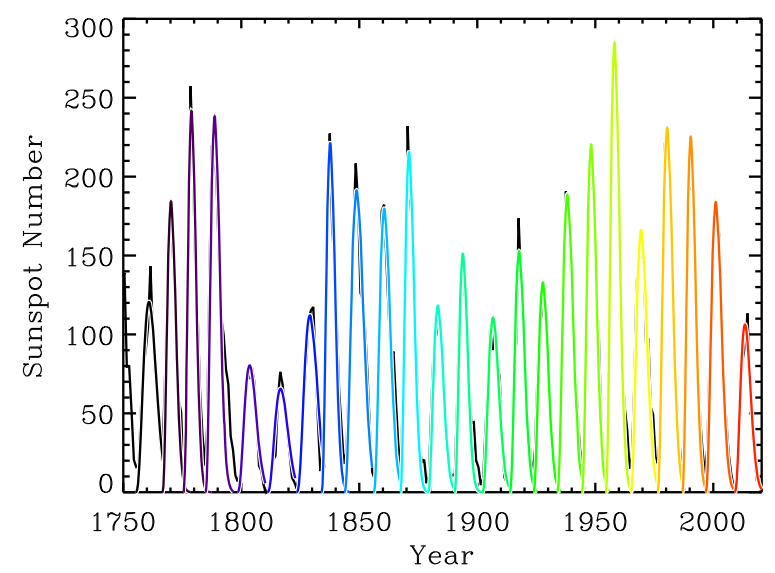

Fig. 3. The time dependence of annually-averaged $S$ (in black) plotted as a function of time. The converged fit for each Solar Cycle is plotted in a color that varies from purple to red going from left to right.

predictions (Fig. 3). These relationships are $A=4.15 \times 10^{-4}$ $S_{\max }^{1.83}$ and $b=15.7 S_{\max }^{-0.265}$ years. A fit to the monthly-mean sunspot number gives the same $A$ but $b$ changes slightly to $15.7 S_{\max }^{-0.272}$ years. This difference disappears when the coefficients in the fits are rounded to two significant digits. These relationships between $A$ and $b$ can be combined to eliminate $S_{\text {max }}$ and produce a relationship between $A$ and $b$.

Because the SODA Index uses F10.7 as the index of solar activity, we repeated the fits with values of annually-averaged F10.7. The minimum value of F10.7 was subtracted before calculating the fits. The fit coefficients are $A_{F}=3.13 \times$ $10^{-4} \mathrm{~F} 10.7_{\max }^{1.99}$ and $b_{F}=13.9 \mathrm{~F} 10.7_{\max }^{-0.267}$ years.

\subsection{Why predict $S$ ?}

The original sunspot number $\left(R_{Z}\right)$ was shown to be a direct proxy of vertical magnetic flux (Stenflo, 2012) and the total magnetic flux (Wang \& Sheeley Jr., 2002), forming a unique reference for solar dynamo models, climate studies, and solar activity predictions. Because $S$ is heavily based on the same input data, $S$ should also be a good proxy of magnetic flux. That means predictions of $S$ are also predictions of the Sun's magnetic field. Sunspot number is also highly correlated with modern quantitative indices such as F10.7, EUV flux, occurrence of flares, and active region areas. 
However, time scales are important. $S$ isn't a good predictor of flares; while short-term predictions of $\boldsymbol{B}$ may be useful for flares but don't help in multi-year forecasts.

\subsection{What else could or should be predicted?}

Sunspot number corresponds to a quantity that covers less than half of the solar surface. Are there quantities that cover larger areas of the Sun that could be used as the predicted variable? One such variable is the magnetic field. Our quantitative knowledge of the solar magnetic field continues to grow, but spans only four sunspot cycles. Other quantitative data with similar (or longer) temporal coverage that could be used to predict Space Weather effects are:

1. F10.7: A direct measure of the effect of the magnetic field on electrons but an indirect measure of the energy input into planetary atmospheres. Tends to be strongly weighted to the active latitudes. Already predicted by several authors.

2. EUV spectral irradiance: The solar extreme ultraviolet spectral irradiance (EUV, reported as a function of wavelength in units of $\mathrm{W} \mathrm{m}^{-2} \mathrm{~nm}^{-1}$ ) corresponds to emissions with wavelengths between $10 \mathrm{~nm}$ and $124 \mathrm{~nm}$. EUV emissions are the source of the bulk of the ionosphere and are well-correlated to the ground-based indices $R_{Z}, S$, and F10.7. If the Sun were a blackbody at an effective temperature of $5770 \mathrm{~K}$ there would be no almost no solar emissions at EUV wavelengths. For example, the ratio of the spectral irradiance of a $5770 \mathrm{~K}$ blackbody at $30.4 \mathrm{~nm}$ to its peak value at a visible wavelength of $500 \mathrm{~nm}$ is $10^{-26}$. Due to the effects of the solar magnetic field this ratio is $10^{-4}$ in the solar spectrum because of the brightness of the He II 304 emission line. The Sun emits EUV radiation over much of the surface and corona, making it a more global quantity than $S$. Continuous measurements of the EUV spectral irradiance exist since 2001 , covering only two relatively weak sunspot cycles, so this is probably insufficient coverage of the possible solar cycle changes. Flares with X-ray irradiances energy covering the known range have occurred in those two cycles. So sufficient data may exist to enable short-term forecasting of the EUV spectral irradiances.

3. X-ray flares: X-ray flare irradiance has been measured since 1965, some five sunspot cycles of coverage. An energy source to the lower thermosphere, flares are responsible for many of the short-term variations in the ionosphere. Heavily weighted to active latitudes. The gross counts of solar flares follow the sunspot number, mostly because flares tend to occur above active regions.

4. Filaments: An indicator of the magnetic field in the corona and chromosphere. The loss of a filament as a coronal mass ejection is the most geoeffective Space Weather source on the Sun. A plot of the filaments in Cartes Synoptiques de la Chromosphère Solaire shows that filaments pretty much cover the Sun during solar maximum. Filaments were used to partition the solar magnetic field to develop a precursor of the next solar cycle by (Tlatov, 2009).

5. Properties of the polar coronal hole and polar magnetic field: The area of the polar coronal hole $(\mathrm{PCH})$ has been examined as a possible precursor of solar activity
(Kirk et al., 2009, 2019, Hess Webber et al., 2014). Although the $\mathrm{PCH}$ area variations are out-of-phase with solar activity that time dependence still includes large annual and semi-annual variations that confuse the interpretation of the data. Worse, the area at minimum does not vary with the polar magnetic field in the few available examples (Kirk et al., 2009).

6. Properties of the polar magnetic field: The strength of the polar magnetic field has been used as a precursor for the last three sunspot cycles. Extending the record using polar faculae may provide a longer baseline of measurements (Schatten \& Pesnell, 1993; Priyal et al., 2014). If the polar field can be predicted then the prediction of following cycles may be possible.

7. Energetic particles from the Sun and magnetosphere: Energetic particles can come directly from a flare or indirectly from a coronal mass ejection or high-speed stream. A prediction of the direct input from flares would simply come from the prediction of the flare. The indirect particles are more difficult as the solar input has to propagate to the Earth and interact with the magnetosphere. This can produce high energy particles but also a complicated geocentric latitude and time dependence.

One plan would be to have mixed timescale predictions:

1. Provide long-term (year-decade) forecasts of the solar activity using precursor-oriented forecasts in combination with flux-transport models.

2. Use short-term forecasts of the EUV spectral irradiance to predict daily to monthly variations of solar output relevant to predicting the ionosphere \& thermosphere.

3. Build models of the solar magnetic field that can forecast the occurrence and strength of solar flares to predict rapid response of ionosphere $\&$ thermosphere as well as radio communication and navigation outages.

4. Have an accurate model of the photoelectrons produced by the absorption of the X-ray and EUV photons.

However, the sunspot number is still our only truly longterm solar activity index. Until we have 4-5 more cycles of EUV spectral irradiance, especially having several cycles that grow to levels of activity similar to Solar Cycle 19, $S$ and F10.7 are still the prediction variables for long-term work.

\section{Predictions of Solar Cycle 24}

At least 105 predictions of the amplitude of Solar Cycle 24 $\left(R_{24}\right)$ were produced before, during, and after solar maximum in February 2014. In this discussion the amplitude will refer to the annually-average sunspot number. For Solar Cycle 24 this is $R_{24}$ and for Solar Cycle 25 this will be $S_{25}$, reflecting the change to Version 2 of the International Sunspot Number. Most of the predictions, individually and categorized, did not produce an accurate forecast of Solar Cycle 24. Only the solar polar field category had a significantly positive skill score (Pesnell, 2018).

But a poor prediction is not "bad" - if what you learn from try $N$ allows you to generate a better try for $N+1$. 


\subsection{What can be learned from ensemble predictions?}

Ensemble predictions place individual predictions in categories to form groups of similar algorithmic output. So long as the algorithms are similar, the ensemble may provide a more accurate forecast as well as an assessment of the algorithm(s). The ensemble can also show when one algorithm is not as effective as another. This differs from ensemble weather forecasts. In that case, you vary the initial conditions consistent with their errors (and possibly the assimilated data within their errors) to see how the forecast changes. By averaging many results you should produce a more accurate forecast. Solar cycle predictions are not typically time integrations of conservation equations (although dynamo models do those integrations.) Here the averaging is over the different algorithms to see if the categories provide a more accurate forecast.

Several studies have examined the accuracy of the ensembles of amplitude predictions for Solar Cycles 20-24. (Vitinskii, 1965, Table 21) lists seven forecasts of the amplitude of Solar Cycle 20, but does not critically compare the methods. McIntosh et al. (1979) discussed 38 predictions of Solar Cycle 21 without categorizing them. Brown (1986) categorized the predictions for Solar Cycle 21 and summarized 31 predictions of Solar Cycle 22 by category. Li et al. (2001) split 63 predictions of Solar Cycle 22 into Groups A (37 predictions that rely solely on solar activity data) and B (26 that use solar activity data along with additional external information such as solargeophysical data). Li et al. (2001) also categorized 48 predictions of Solar Cycle 23 into the same Groups A (31) and B (17). Solar Cycle 23 appears to have been the first cycle where an official panel produced a consensus forecast (Joselyn et al., 1997). The panel considered 28 predictions of the amplitude, dividing them into six categories (Climatology, Even/Odd, Neural network, Precursor, Recent climatology, and Spectral) but not showing the numbers in each category.

Pesnell $(2016,2018)$ examined the ensemble of predictions for Solar Cycle 24. He followed the Solar Cycle 24 Consensus panel and replaced the Even/Odd category of the Solar Cycle 23 panel with the Dynamo Model category (Biesecker \& The Solar Cycle 24 Prediction Panel, 2007). Another ensemble analysis of Solar Cycle 24 predictions is described by Petrovay (2020). They divided 23 predictions into three categories: precursor methods, model-based methods (consistent with the dynamo category), and extrapolation methods (a combination of the climatological, spectral, and neural net categories).

\subsection{Climatological or statistical methods}

Climatological or statistical forecasts assume that the future of a system can be determined from the statistical properties of the past. One example is that $R_{24}$ will be the average of all observed maxima, $R_{\mathrm{ave}}=115 \pm \sigma_{0}$, where $\sigma_{0}=40$ provides a standard error estimate (Pesnell, 2008). This average is an important comparison for all other predictions.

This category had a large spread in values, with the largest and smallest predictions of $R_{24}$. The values were clustered near the climatological average rather than the actual, below-average, value. Pesnell (2018) found that the climatological predictions of Solar Cycle 24 were less accurate than those in the precursor category. This agrees with Brown (1986) and Li et al. (2001), who concluded that methods using a single source of information gave more widely varying predictions for Solar Cycles 21-24.

This category has now done poorly in compilations of predictions for Solar Cycles 21-24. Perhaps splitting into subcategories, to better understand their predictive skill, might make these predictions more useful.

\subsection{Neural nets or machine learning}

Neural nets (NN, also ML and $\mathrm{AI}$ ) forecasts are derived from nonlinear, statistical algorithms that determine and model complex relationships between inputs and outputs to find patterns in the data that can be extrapolated. With the increase in computer capabilities and access to ever-larger datasets, neural net predictions have become quite common. An analysis of sunspot number is often a first project in Machine Learning (see, for example, Dancho \& Keydana, 2018).

The six neural network forecasts for Solar Cycle 24 had a large dispersion, and the four later entries were for smaller amplitudes than the first two. The seven existing NN and ML predictions of SC 25 have an average of $S_{25}=100 \pm 45$.

My concern with these forecasts is that they are essentially climatological with a hidden layer mystifying the reasons for the correlations. They may find correlations we hadn't anticipated but for Solar Cycle 24 they did no better than hand-crafted correlations. One interesting prediction of Solar Cycle 25 used a feed-forward $\mathrm{NN}$ to predict the sunspot butterfly diagram (Covas et al., 2019). The authors then summed over latitude to produce a forecast of a very low amplitude for Solar Cycle 25 as well as the shape of Solar Cycle 25. Such predictions might be improved if the magnetic butterfly diagram was used. This would include information about the polar regions in the analysis. Although the sum over latitude to calculate the active region contribution would have to omit the poleward surges and poles, a sum over latitude would include the long-lived magnetic fields near the poles.

\subsection{Dynamo models}

The Dynamo Model category was new in Solar Cycle 24. Although it had the second fewest contributions, the standard deviation of the predictions was large enough to include the actual amplitude of Solar Cycle 24. The last 12 years have seen a lot of work in dynamo models to enable better predictions. More accurate models of the erupting magnetic field, necessary to allow them to be compared with measurements, are described by Yeates \& Muñoz-Jaramillo (2013). Some early work in assimilating data into a realistic model was described by Svedin et al. (2013) and Svedin (2013). Unlike the troposphere, we have limited information about the interior of the Sun and no in situ data about conditions between the photosphere and temperature minimum at the top of the chromosphere.

\subsection{Precursors}

Precursor predictions are the leading indicators of solar activity and remain the most common category of predictions. These predictions use multiple sources, have smaller scatter, and tend to group around the actual amplitude of the upcoming maximum (Pesnell, 2016). This led to the common wisdom that precursors were superior, which seems to be valid in Solar 
Cycle 24. This category had the largest number of predictions of Solar Cycle 24.

One class of precursor predictions combine geomagnetic activity and solar activity into a geomagnetic precursor pair (Ohl \& Ohl, 1979; Feynman, 1982; Hathaway \& Wilson, 2006; Pesnell, 2014). Another class that combines the solar polar field with an index of solar activity was first described by Schatten et al. (1978). Geomagnetic and solar polar precursor predictions were compared for Solar Cycle 22 by Layden et al. (1991), who found the geomagnetic precursors were more reliable than the polar field precursor because of the paucity of historic solar polar data. This was confirmed for Solar Cycle 23 by Hathaway et al. (2001). A weighted combination of precursor predictions was recommended by Hathaway et al. (1999) to improve the forecast of Solar Cycle 23. The combined prediction was a little lower than the consensus panel value, but was still above the actual maximum.

Some of this over-estimate may come from the sensitivity of geomagnetic precursors to the long decay of Solar Cycle 23. One common algorithm removes the current cycle's activity from the geomagnetic index. In the absence of other information, if that removal is incorrect then so is the precursor prediction (Pesnell, 2014).

Another precursor method uses the number of spotless days near solar minimum to predict the upcoming maximum. Pesnell (2012) showed how marking days in $R_{Z}$ without observations with zeroes caused this method to perform poorly in Solar Cycle 24 . This was corrected in $S$. Helal \& Galal (2013) analyzed the effect of using $S$ instead of $R_{Z}$ on a spotless days prediction of Solar Cycle 25. They produced a prediction of $S_{25}=170$. This prediction should be updated as we now know the annual number of spotless days in 2019, which appears to be the peak in this minimum.

Figure 4 shows the correlation plot between the maximum annual number of spotless days as the abscissa and the maximum annual sunspot number as the ordinate. The significance of this correlation can be estimated from the correlation coefficient $(r=-0.367)$ and number of points (18). Using the two-sided test, there is a $13 \%$ chance that the correlation is spurious. A linear fit of $S_{n}=242-0.33 \mathrm{SP}_{n}$ gives $S_{25}=150 \pm 20$ for $\mathrm{SP}_{25}=273$. The fit assumed there were errors in both variables. This prediction requires data one year past solar minimum to guarantee that the maximum in spotless days has passed.

Petrovay (2020) concluded that the solar polar field precursor was consistent and that precursor methods were superior to their listed extrapolation methods, which are a combination of the climatological, spectral, and neural net categories.

Solar polar precursors are less sensitive to the phasing of solar minimum because they use a peaking leading indicator instead of a vanishing one. They are discussed in Section 5 .

\subsection{Recent climatology}

The Recent Climatology class was very sparse in Solar Cycle 24 predictions. Only four entries by two authors, with a tight grouping well above the actual value and the average. This category emphasizes recent behavior over the long-term. Solar Cycles 21-23 had a general downward trend and any persistence or Even-Odd forecast would be unable to break that trend. Only when the full variability of the solar dynamo is captured

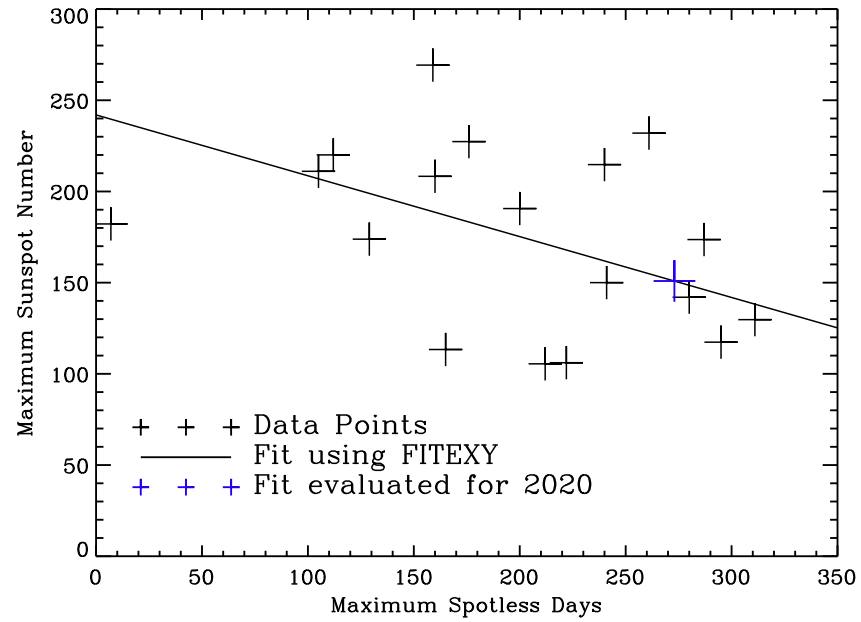

Fig. 4. The maximum annual number of spotless days in each intercycle interval vs. maximum annual sunspot number of the following solar cycle. Values for Solar Cycles 1-24 are shown as black plus signs. The solid line is a linear fit to the data and the blue " + " is the prediction that $S_{25}=150$ based on using a spotless day count of 273 in 2019 in that fit.

can a climatological prediction be expected to make an accurate prediction.

Some naïve predictions for Solar Cycle 25 in this category were explored by Pesnell (2018). No predictive skill was found in those studies. The estimated forecast error approached the limit of $\sqrt{2} \times \sigma_{0}$, where $\sigma_{0}$ is the standard deviation of all maxima from the climatological average, that indicates the forecasts are simply random numbers with no predictive skill.

\subsection{Spectral}

Spectral forecasts look for invariant quantities in a Fourier analysis of the sunspot time series. Invariants could include frequencies whose amplitudes are either conserved or can be represented with a simple time dependence. Autoregressive forecasts, wavelet-based forecasts, and predictions based on the motion of the solar system baricenter were placed in this category. Some of the nonlinear time series analysis models that are based on an explicit dynamo model equation were placed in this category. The 24 forecasts in this category tended to predict that Solar Cycle 24 will have slightly below-average activity. One standard deviation from the mean of this category included both the long-term average and the actual amplitude of Solar Cycle 24.

Spectral methods appear to be very effective because the fit looks smooth. A simple Fourier fit to $S$ (after inverting every other cycle as described by Bracewell, 1953) is an excellent reproduction of the data. You can then put any dates into the fitting function and get an extrapolation of $S$. Unfortunately, that extrapolation is a reprise of the fit. You can get no new behavior, such as a maximum that differs from the fitted data, from the fit. If you include the Maunder Minimum (from 1645 to 1715 ) in your fit, then roughly every 400 years you will predict another Grand Minimum.

Long-term predictions can use Fourier analysis of actual data and then make assumptions about the variation of the 
Table 1. Examples of Solar Cycle 25 predictions.

\begin{tabular}{lccc}
\hline Assumption & $S_{25}$ & Date of max. & Reference \\
\hline SODA index & $135 \pm 40$ & $2025.2 \pm 1.5$ & Pesnell \& Schatten (2018) \\
SODA index (update) & $125 \pm 40$ & $2024.6 \pm 1.5$ & Current work \\
Cycle $N+1=\langle N\rangle$ & $180 \pm 60$ & $2025.4 \pm 1.2$ & Pesnell (2018) \\
Cycle $N+1=$ Cycle $N-1$ & - & Pesnell (2018) \\
Cycle $N+1=$ Cycle $N$ & $180 \pm 85$ & - & Pesnell (2018) \\
Spotless days & $115 \pm 70$ & - & Current work, Section 4.5 \\
\hline
\end{tabular}

Fourier coefficients to extrapolate the future. Any extrapolation has to report an error that increases with time. The error may be small at the beginning of the interval, but the uncertainty grows without bound until another calibration point can be inserted. All long-term extrapolation algorithms have this problem.

\section{Success of polar field predictions}

Polar field precursors (like SODA) have done a reasonable job of predicting the peak of four cycles, often before minimum (Pesnell \& Schatten, 2018).

The magnetic field near both poles goes through zero near solar maximum and reaches its largest magnitude near solar minimum. Its averaged value near solar minimum has been used as a precursor of solar activity (Schatten \& Pesnell, 1993; Schatten, 2005; Svalgaard et al., 2005; Pesnell \& Schatten, 2018; Bisoi et al., 2020).

Figure 4 shows the variation of the solar polar magnetic field since the Wilcox Solar Observatory began regular observations in $1976 .^{3}$ The magnetic field measurements were averaged over six-month intervals. The northern (blue) and sign-reversed southern (red) magnetic field are shown separately, along with their difference (green). The difference is a better indicator of the average global magnetic field. The modulation of the polar field sampling caused by tilt of the solar rotation axis with respect to the ecliptic plane is greatly reduced in this smoothed and averaged data.

The time dependence of the polar magnetic fields and their usefulness as a precursor have been examined (MuñozJaramillo et al., 2013; Upton \& Hathaway, 2018). These fields were also used to drive an assimilative model of the solar cycle that made a prediction of a low amplitude for Solar Cycle 24 (Choudhuri et al., 2007).

\section{Solar Cycle 25 is coming!}

Published predictions of Solar Cycle 25 span from an average amplitude to small or absent (Upton et al., 2019). It appears that predictions of Solar Cycle 25 will again have a large spread of values. But the largest prediction amplitude is less than $\sigma_{0}$ above the average while the largest prediction was almost $2 \sigma_{0}$ in Solar Cycle 24 .

Table 1 shows several predictions of annually-averaged $S_{25}$ by this author. For example, Pesnell (2018) calculated the climatological average of $S\left(S_{\text {ave }}=180\right)$, the most robust statistical forecast, the value of $\sigma_{0}=60$, and the timing of

\footnotetext{
${ }^{3}$ http://wso.stanford.edu/Polar.html
}

extrema in $S$. He also showed that two recent climatology predictions were essentially producing random results.

The SODA Index (update) in Table 1 predicts a smaller amplitude of Solar Cycle 25, and the timing of maximum moved earlier. The latter is caused by the new shape function described in Section 3.2 rising more quickly than the fit in Hathaway et al. (1994).

\section{Conclusions}

Solar cycle predictions are an interesting and practical area of research. Only by making and analyzing these predictions will we build accurate models of the solar dynamo. The amplitude predictions of Solar Cycle 24 spanned all possible historical values, from vanishing to unprecedented levels of activity. Only the solar polar field category was consistent in predicting the amplitude of Solar Cycle 24.

1. An ensemble analysis of the many predictions of Solar Cycle 24 shows consistency with the category analyses of earlier authors. The climatological or statistical forecasts have performed poorly in four sunspot cycles. Precursor predictions have fared better, but many of them require information close to or spanning solar minimum. The one new category, dynamo models, generated more effort in flux-transport models as well as full-blown convection zone models.

2. We should give less emphasis on climatological or statistical predictions. Few seem to work. This may include neural nets and machine learning algorithms that focus only on data involving active regions. But so far 16 climatological or statistical forecasts of Solar Cycle 25 have been published. And, once again, they span the range of the predictions.

3. A variable suitable for predicting solar cycle amplitudes should include as much of the past variability as possible. Otherwise, local trends can dominate the prediction. Solar Cycles 19-24 roughly decreased in amplitude, meaning the Space Age has only seen one cycle that increased in activity (SC 20-21). How does that limit our understanding of the dynamo? Quantitative measurements of the global solar magnetic field span an even smaller time period.

4. Predictions of Solar Cycle 25 have a smaller spread than did those of Solar Cycle 24 and tend to be grouped around the same amplitude as SC 24.

5. Even as we converge towards an ensemble prediction of the amplitude, the timing of the next solar maximum should also be considered. Although the amplitude and 


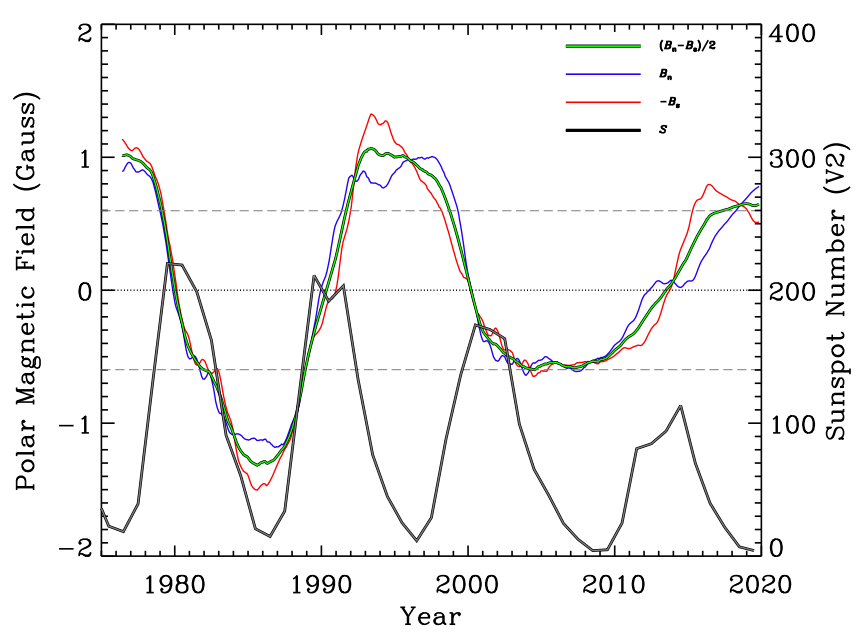

Fig. 5. The polar magnetic field from the Wilcox Solar Observatory. The northern and the negative of the southern magnetic field are shown in blue and red, respectively, along with their difference in green. The oscillations caused by the tilt of the solar rotation axis to the ecliptic are greatly suppressed.

timing predictions are linked by the Waldmeier effect, where higher amplitudes is correlated with shorter cycles, they can be done independently.

6. Moving the date of accurate predictions to ever-earlier times before solar minimum or extending the predictions to include multiple sunspot cycles has been requested by the users of the predictions. Until we can accurately predict the next cycle the best we can do is embrace $\left\langle S_{\max }\right\rangle=180 \pm 60$ as the prediction of following cycles. Families of predicted cycles can be generated and used as conditions in Monte Carlo explorations of the future. Creating earlier accurate predictions means finding precursors with a valid value before minimum. The solar polar field tends to reach its peak soon after solar maximum (Fig. 5) and the scatter about that value is one contributor to the error estimate.

7. The success of the polar field precursor predictions shows that we should determine what information is missing in our understanding of the solar polar regions. This region of the Sun is where the solar dynamo manifests during solar minimum. Understanding its variation is as important as understanding the sunspot record. The polar crown filaments are the only place where the magnetic field of the new cycle merges with and removes the field of the previous cycle (Karna et al., 2017). This may account for their longevity and orientation. The polar coronal hole is still a fuzzy object that refuses to be accurately measured (Hess Webber et al., 2014; Karna et al., 2014; Kirk et al., 2019).

Unfortunately, ham radio operators may be in for another disappointing 10 years of poor HF propagation. The polar magnetic field shows that Solar Cycle 25 will peak in late 2024 or early 2025 with a slightly higher amplitude than Solar Cycle 24!

Acknowledgements. This work was supported by NASA's Solar Dynamics Observatory at the Goddard Space Flight Center. The International Sunspot Number (Version 2) data were obtained from the Solar Influences Data Center (SIDC, http://sidc.oma.be/silso/datafiles), as the monthly samples of the 13-month running average (SN_ms_tot_V2.0.csv) and the annual average (SN_y_tot_V2.0.csv). Values of Version 1 of the International Sunspot Number between 01 Jan 1850 and 31 May 2015 were obtained from the archival website at SIDC (http://www.sidc.be/silso/DATA/dayssnv0.dat). Adjusted F10.7 (normalized to $1 \mathrm{AU}$ ) values are courtesy of the Dominion Radio Astronomy Observatory, Penticton, British Columbia, Canada, and were downloaded from ftp:// ftp.geolab.nrcan.gc.ca/data/solar_flux/daily_flux_values/fluxtable.txt. The Wilcox Solar Observatory polar field strengths came from http://wso.stanford.edu/Polar.html and are courtesy of J. T. Hoeksema. The Wilcox Solar Observatory is currently supported by NASA. The editor thanks two anonymous reviewers for their assistance in evaluating this paper.

\section{References}

Biesecker D., The Solar Cycle 24 Prediction Panel. 2007. Consensus statement of the Solar Cycle 24 prediction panel, released March 2007. URL https://www.swpc.noaa.gov/SolarCycle/SC24/.

Bisoi SK, Janardhan P, Ananthakrishnan S. 2020. Another mini solar maximum in the offing: A prediction for the amplitude of Solar Cycle 25. J Geophys Res: Space Phys 125: e2019JA027508.

Bracewell RN. 1953. The sunspot number series. Nature 171(4354): 649-650. https://doi.org/10.1038/171649a0.

Brown G. 1986. Working group "A" report: Long-term solar activity predictions. In: Solar-terrestrial predictions, Simon A, Heckman G, Shea MA (Eds.), pp. 1-7.

Choudhuri A, Chatterjee P, Jiang J. 2007. Predicting Solar Cycle 24 with a solar dynamo model. Phys Rev Lett 98(131): 103. https://doi.org/10.1103/PhysRevLett.98.131103.

Clette F, Lefèvre L. 2016. The new sunspot number: Assembling all corrections. Sol Phys 291: 2629-2651. https://doi.org/10.1007/ s11207-016-1014-y.

Clette F, Svalgaard L, Vaquero JM, Cliver EW. 2014. Revisiting the sunspot number. A 400-year perspective on the solar cycle. Space Sci Rev 186: 35-103. https://doi.org/10.1007/s11214-014-0074-2.

Cook AF. 1949. On the mathematical characteristics of sunspotvariations. J Geophys Res 54(4): 347-354. https://doi.org/10.1029/ JZ054i004p00347.

Covas E, Peixinho N, Fernandes J. 2019. Neural network forecast of the sunspot butterfly diagram. Sol Phys 294(3): 24. https://doi.org/ 10.1007/s11207-019-1412-z.

Covington AE. 1969. Solar radio emission at $10.7 \mathrm{~cm}, 1947-1968$. $J$ R Astron Soc Can 63: 125-132.

Dancho M, Keydana S. 2018. TensorFlow for R: Predicting sunspot frequency with Keras. https://blogs.rstudio.com/tensorflow/posts/ 2018-06-25-sunspots-1stm/.

Feynman J. 1982. Geomagnetic and solar wind cycles, 1900-1975. J Geophys Res 87: 6153-6162.

Hathaway DH, Wilson RM. 2006. Geomagnetic activity indicates large amplitude for sunspot cycle 24. Geophys Res Lett 33(L18): 101. https://doi.org/10.1029/2006GL027053.

Hathaway DH, Wilson RM, Reichman EJ. 1994. The shape of the sunspot cycle. Sol Phys 151: 177-190. https://doi.org/10.1007/ BF00654090.

Hathaway DH, Wilson RM, Reichman EJ. 1999. A synthesis of solar cycle prediction techniques. J Geophys Res 104: 375-388. https://doi.org/10.1029/1999JA900313. 
Hathaway DH, Wilson RM, Reichmann EJ. 2001. Status of cycle 23 forecasts. Washington DC Am Geophys Union Geophys Monogr Ser 125: 195-200. https://doi.org/10.1029/GM125p0195.

Helal HR, Galal A. 2013. An early prediction of the maximum amplitude of the solar cycle 25. J Adv Res 4(3): 275-278. https://doi.org/10.1016/j.jare.2012.10.002.

Hess Webber SA, Karna N, Pesnell WD, Kirk MS. 2014. Areas of polar coronal holes from 1996 through 2010. Sol Phys 289: 40474067. https://doi.org/10.1007/s11207-014-0564-0.

Joselyn J, Anderson J, Coffey H, Harvey K, Hathaway D, et al. 1997. Panel achieves consensus prediction of Solar Cycle 23. EOS Trans $A G U$ 78: 205-212. https://doi.org/10.1029/97EO00136.

Karna N, Hess Webber SA, Pesnell WD. 2014. Using polar coronal hole area measurements to determine the solar polar magnetic field reversal in Solar Cycle 24. Sol Phys 289: 3381-3390. https://doi. org/10.1007/s11207-014-0541-7.

Karna N, Zhang J, Pesnell WD. 2017. The formation and maintenance of the dominant southern polar crown cavity of cycle 24 . Astrophys $J$ 835(2): 135. https://doi.org/10.3847/1538-4357/835/2/135.

Kirk MS, Pesnell WD, Young CA, Hess Webber SA. 2009. Automated detection of EUV polar coronal holes during Solar Cycle 23. Sol Phys 257: 99-112. https://doi.org/10.1007/s11207009-9369-y.

Kirk MS, Pesnell WD, Arge C. 2019. A comprehensive assessment of EUV polar coronal holes: 1996-2018. In: American Astronomical Society Meeting Abstracts \#234, Vol. 234 of American Astronomical Society Meeting Abstracts, $125.01 \mathrm{p}$.

Layden AC, Fox PA, Howard JM, Sarajedini A, Schatten KH. 1991. Dynamo-based scheme for forecasting the magnitude of solar activity cycles. Sol Phys 132: 1-40. https://doi.org/10.1007/ BF00159127.

Li KJ, Yun HS, Gu XM. 2001. On long-term predictions of the maximum sunspot numbers of solar cycles 21 to 23. A\&A 368: 285-291. https://doi.org/10.1051/0004-6361:20000547.

McIntosh P, Brown G, Buhmann R, Clark T, Fougere P, Hunter H, Lincoln J, Sargent I, Timothy J, Lin Y. 1979. Long-term solar activity predictions. In: NOAA solar-terrestrial predictions proceedings, Donnelly R (Ed.), Vol. 2, pp. 246-257.

Muñoz-Jaramillo A, Balmaceda LA, DeLuca EE. 2013. Using the dipolar and quadrupolar moments to improve solar-cycle predictions based on the polar magnetic fields. Phys Rev Lett 111(041): 106. https://doi.org/10.1103/PhysRevLett.111.041106.

Ohl AI, Ohl GI. 1979. A new method of very long-term prediction of solar activity. In: Solar-Terrestrial Predictions Proceedings, Donnelly R (Ed.), Vol. 2, NOAA/Space Environment Laboratory, pp. 258-263.

Pesnell WD. 2008. Predictions of Solar Cycle 24. Sol Phys 252: 209220. https://doi.org/10.1007/s11207-008-9252-2.

Pesnell WD. 2012. Solar cycle predictions (invited review). Sol Phys 281: 507-532. https://doi.org/10.1007/s11207-012-9997-5.

Pesnell WD. 2014. Predicting Solar Cycle 24 using a geomagnetic precursor pair. Sol Phys 289: 2317-2331. https://doi.org/10.1007/ s11207-013-0470-x.

Pesnell WD. 2016. Predictions of Solar Cycle 24: How are we doing? Space Weather 14: 10-21. https://doi.org/10.1002/ 2015SW001304.

Pesnell WD. 2018. Effects of version 2 of the International Sunspot Number on naïve predictions of Solar Cycle 25. Space Weather 16: 1997-2003. https://doi.org/10.1029/2018SW002080.

Pesnell WD, Schatten KH. 2018. An early prediction of the amplitude of Solar Cycle 25. Sol Phys 293: 112. https://doi.org/ 10.1007/s11207-018-1330-5.
Petrovay K. 2020. Solar cycle prediction. Living Rev Sol Phys 17(1): 2. https://doi.org/10.1007/s41116-020-0022-z.

Priyal M, Banerjee D, Karak BB, Muñoz-Jaramillo A, Ravindra B, Choudhuri AR, Singh J. 2014. Polar network index as a magnetic proxy for the solar cycle studies. Astrophys J Lett 793(1): L4. https://doi.org/10.1088/2041-8205/793/1/L4.

Russell CT, Mewaldt RA, Luhmann JG, Mason GM, von Rosenvinge TT, et al. 2013. The very unusual interplanetary coronal mass ejection of 2012 July 23: A blast wave mediated by solar energetic particles. Astrophys J 770: 38. https://doi.org/ 10.1088/0004-637X/770/1/38.

Schatten KH. 2005. Fair space weather for solar cycle 24. Geophys Res Lett 32(L21): 106. https://doi.org/10.1029/2005GL024363.

Schatten KH, Pesnell WD. 1993. An early solar dynamo prediction: Cycle $23 \sim$ cycle 22. Geophys Res Lett 20: 2275-2278. https://doi. org/10.1029/93GL02431.

Schatten KH, Scherrer PH, Svalgaard L, Wilcox JM. 1978. Using Dynamo Theory to predict the sunspot number during Solar Cycle 21. Geophys Res Lett 5: 411-414. https://doi.org/10.1029/ GL005i005p00411.

Solomon SC, Woods TN, Didkovsky LV, Emmert JT, Qian L. 2010. Anomalously low solar extreme-ultraviolet irradiance and thermospheric density during solar minimum. Geophys Res Lett 37: L16103. https://doi.org/10.1029/2010GL044468.

Stenflo JO. 2012. Basal magnetic flux and the local solar dynamo. A\&A 547: A93. https://doi.org/10.1051/0004-6361/201219833.

Stewart JQ, Panofsky HAA. 1938. The mathematical characteristics of sunspot variations. Astrophys J 88: 385. https://doi.org/10.1086/ 143994.

Sun X, Bobra MG, Hoeksema JT, Liu Y, Li Y, Shen C, Couvidat S, Norton AA, Fisher GH. 2015. Why is the great solar active region 12192 flare-rich but CME-poor? Astrophys J Lett 804: L28. https://doi.org/10.1088/2041-8205/804/2/L28.

Svalgaard L, Cliver E, Kamide Y. 2005. Cycle 24: The smallest sunspot cycle in 100 years? Geophys Res Lett 32: L01104. https://doi.org/10.1029/2004GL021664.

Svedin A. 2013. Nonlinear data assimilation: Towards a prediction of the solar cycle. Ph.D. thesis, Columbia University, New York.

Svedin A, Cuéllar MC, Brandenburg A. 2013. Data assimilation for stratified convection. Mon Not R Astron Soc 433: 2278-2285. https://doi.org/10.1093/mnras/stt891.

Tapping KF, Charrois DP. 1994. Limits to the accuracy of the 10.7 $\mathrm{cm}$ flux. Sol Phys 150: 305-315. https://doi.org/10.1007/ BF00712892.

Tlatov AG. 2009. The minimum activity epoch as a precursor of the solar activity. Sol Phys 260(2): 465-477. https://doi.org/10.1007/ s11207-009-9451-5.

Upton LA, Hathaway DH. 2018. An updated Solar Cycle 25 prediction with AFT: The modern minimum. Geophys Res Lett 45: 8091-8095. https://doi.org/10.1029/2018GL078387.

Upton L, Biesecker D, The Solar Cycle 25 Prediction Panel. 2019. Solar Cycle 25 predictions. URL https://www.swpc.noaa.gov/ sites/default/files/images/u59/10\%20Lisa\%20Upton\%20Official. pdf.

Vitinskii YI. 1965. Solar-activity forecasting. In: Vol. F-289 of Technical translation, NASA, Scientific and Technical Information Office, Washington, DC.

Wang Y-M, Sheeley NR Jr. 2002. Sunspot activity and the long-term variation of the Sun's open magnetic flux. J Geophys Res: Space Phys 107(A10): SSH 10-1-SSH 10-15. https://doi.org/10.1029/ 2001JA000500. https://agupubs.onlinelibrary.wiley.com/doi/abs/ 10.1029/2001JA000500. 
Weatherley TF. 1980. Sunspots. What Do They Mean? - Your guess is an good as mine. 73 Magazine, pp. 28-30. https://archive. org/stream/73-magazine-1980-04/04_April_1980\#page/n29/mode/ 2up.
Wolf R. 1861. Mittheilungen über die Sonnenflecken XII. Astron Mitt Eidgenüssischen Sternwarte Zurich 2: 41-82.

Yeates AR, Muñoz-Jaramillo A. 2013. Kinematic active region formation in a three-dimensional solar dynamo model. Mon Not $R$ Astron Soc 436: 3366-3379.

Cite this article as: Pesnell WD 2020. Lessons learned from predictions of Solar Cycle 24. J. Space Weather Space Clim. 10, 60. https://doi. org/10.1051/swsc/2020060. 